\title{
Risk of Adverse Outcomes in Hospitalized Patients With Autoimmune Disease and COVID-19: A Matched Cohort Study From New York City
}

\author{
Adam S. Faye ${ }^{1}$, Kate E. Lee ${ }^{2}$, Monika Laszkowska ${ }^{3}$, Judith Kim³ ${ }^{3}$ John William Blackett ${ }^{3}$, \\ Anna S. McKenney ${ }^{4}$, Anna Krigel ${ }^{3}$, Jon T. Giles ${ }^{5}$, Runsheng Wang ${ }^{5}$, Elana J. Bernstein ${ }^{5}$, \\ Peter H.R. Green ${ }^{3}$, Suneeta Krishnareddy ${ }^{3}$, Chin Hur ${ }^{3}$, and Benjamin Lebwohl ${ }^{3}$
}

\begin{abstract}
Objective. To examine the effect of autoimmune (AI) disease on the composite outcome of intensive care unit (ICU) admission, intubation, or death from COVID-19 in hospitalized patients.

Methods. Retrospective cohort study of 186 patients hospitalized with COVID-19 between March 1, 2020, and April 15, 2020 at NewYork-Presbyterian Hospital/Columbia University Irving Medical Center. The cohort included 62 patients with AI disease and 124 age- and sex-matched controls. The primary outcome was a composite of ICU admission, intubation, and death, with secondary outcome as time to in-hospital death. Baseline demographics, comorbidities, medications, vital signs, and laboratory values were collected. Conditional logistic regression and Cox proportional hazards regression were used to assess the association between AI disease and clinical outcomes.

Results. Patients with AI disease were more likely to have at least one comorbidity $(87.1 \%$ vs $74.2 \%$, $P=0.04)$, take chronic immunosuppressive medications $(66.1 \%$ vs $4.0 \%, P<0.01)$, and have had a solid organ transplant $(16.1 \%$ vs $1.6 \%, P<0.01)$. There were no significant differences in ICU admission $(13.7 \%$ vs $19.4 \%, P=0.32)$, intubation $(13.7 \%$ vs $17.7 \%, P=0.47)$, or death $(16.1 \%$ vs $14.5 \%, P=0.78)$. On multivariable analysis, patients with AI disease were not at an increased risk for a composite outcome of ICU admission, intubation, or death $\left(\mathrm{OR}_{\text {adj }} 0.79,95 \% \mathrm{CI} 0.37-1.67\right)$. On Cox regression, AI disease was not associated with in-hospital mortality $\left(\mathrm{HR}_{\text {adj }} 0.73,95 \%\right.$ CI $\left.0.33-1.63\right)$.

Conclusion. Among patients hospitalized with COVID-19, individuals with AI disease did not have an increased risk of a composite outcome of ICU admission, intubation, or death.
\end{abstract}

Key Indexing Terms: autoimmune disease, COVID-19, immunosuppression, SARS-CoV-2

This work was supported by the National Institutes of Health (T32 DK083256 to ASF, K23AR075112 to EJB) and The Louis and Gloria Flanzer Philanthropic Trust (to BL).

'A.S. Faye, MD, MS, Department of Medicine, Division of Digestive and Liver Diseases, NewYork-Presbyterian/Columbia University Irving Medical Center, Department of Medicine, Henry D. Janowitz Division of Gastroenterology, Mount Sinai Hospital; ${ }^{2}$ K.E. Lee, BA, Vagelos College of Physicians and Surgeons, Columbia University; ${ }^{3}$ M. Laszkowska, MD, MS, J. Kim, MD, J.W. Blackett, MD, A. Krigel, MD, MS, P.H. Green, MD, S. Krishnareddy, MD, MS, C. Hur, MD, MPH, B. Lebwohl, MD, MS, Department of Medicine, Division of Digestive and Liver Diseases, NewYork-Presbyterian/Columbia University Irving Medical Center; ${ }^{4}$ A.S. McKenney, MD, PhD, MPH, Department of Radiology, NewYork-Presbyterian/Weill Cornell Medical College; 5 J.T. Giles, MD, MPH, R. Wang, MD, MHS, E.J. Bernstein, MD, MSc, Department of Medicine, Division of Rheumatology, NewYork-Presbyterian/Columbia University Irving Medical Center, New York, New York, USA.

The authors report no conflicts of interest.

Address correspondence to Dr. A.S. Faye, Present/Levison Advanced IBD Fellow, The Susan and Leonard Feinstein IBD Center, The Dr Henry D. Janowitz Division of Gastroenterology, Icahn School of Medicine at Mount Sinai, New York NY, 1468 Madison Ave, Annenberg RM 5-12, New York, NY 10029.Email:adam.faye@mountsinai.org.

Accepted for publication October 8, 2020.
In January 2020, severe acute respiratory syndrome coronavirus 2 (SARS-CoV-2) was isolated and identified as the novel pathogen causing the coronavirus disease 2019 (COVID-19) ${ }^{1}$. In the following months, SARS-CoV-2 spread rapidly to over 200 countries and has spanned the clinical spectrum of disease, ranging from asymptomatic infection to respiratory failure and death ${ }^{2,3,4,5}$. Although several cohort studies have presented characteristics and outcomes for patients with COVID-192,5,6,7,8,9,10, few studies have focused on the impact on those hospitalized who also had autoimmune (AI) disease $\mathrm{e}^{11,12,13,14,15,16,17}$.

It has been suggested that a hyperinflammatory response to COVID-19 (cytokine storm) may lead to a more severe disease course $^{18}$. Accordingly, a retrospective study of 150 COVID-19 cases from Wuhan, China found that increased levels of inflammatory markers, such as ferritin and IL-6, were associated with an increase in overall mortality ${ }^{3}$. Since patients with AI disease have underlying immune dysregulation, it is important to examine how COVID-19 affects their risk of adverse outcomes, including those on chronic immunosuppression.

In a recent matched cohort study by D'Silva, et al, the authors examined adverse outcomes among COVID-19 patients with AI disease and found similar rates of hospitalization and mortality, 
but an increased risk of respiratory failure among patients with AI disease ${ }^{11}$. However, only a small number of patients with AI disease $(n=23)$ were hospitalized, which limits the evaluation of these outcomes. As such, we aimed to analyze a large cohort of hospitalized patients with both AI disease and COVID-19 in order to further assess differences in hospital presentation and clinically relevant outcomes, such as intubation, intensive care unit (ICU) admission, and death.

\section{MATERIALS AND METHODS}

Matched cohort study design. We conducted a retrospective cohort study examining patients, both cases and controls $\geq 18$ years, who presented to NewYork-Presbyterian Hospital/Columbia University Irving Medical Center from March 1, 2020, to April 15, 2020. Only patients found to be positive for SARS-CoV-2 on reverse transcriptase PCR (RT-PCR) were included for analysis. AI disease, including immune-mediated inflammatory diseases (IMID) such as inflammatory bowel disease and spondyloarthritis, was initially identified based on the International Classification of Diseases, 10th revision, criteria from all hospitalized patients with COVID-19 during this time period, with records that were reviewed manually for confirmation. Patients were then classified based on the presence or absence of AI disease (all included AI diseases are presented in Supplementary Table 1, available from the authors on request).

As part of our matched cohort study, each patient with AI disease was binned with all non-AI disease age- and sex-matched controls. Two controls were chosen for each patient with AI disease using a random number generator (matched 2:1, control: AI). All data elements were then manually retrieved from the electronic health record (EHR) to ensure accuracy.

Primary outcome. The primary outcome was a composite of adverse events comprising death, intubation, or admission to an ICU. The secondary outcome was the time to in-hospital death.

Covariables and exposures of interest. Demographics collected included age, sex, race, and ethnicity. Additional exposure factors, such as BMI, smoking status, and prior solid organ transplant, were ascertained. Comorbidities relevant to COVID-19 [i.e., hypertension (HTN), chronic obstructive pulmonary disease, asthma, interstitial lung disease, DM, chronic kidney disease $(\mathrm{CKD})$, coronary artery disease, heart failure, or active malignancy $]^{2,5,19}$ were recorded, then subsequently categorized based on the number of underlying comorbid conditions $(0,1, \geq 2)$. Home medications were manually reviewed and were only classified as "home medications" if used for at least 1 month and continued prior to admission. Medications that were initiated for suspected/confirmed COVID-19 were not included as "home medications." Presenting symptoms, vital signs, and lab values, as well as inpatient treatments, were also obtained from the EHR.

Statistical approach. When comparing baseline characteristics and individual outcomes (intubation, ICU, death) between patients with AI disease and controls, we used the Cochran-Mantel-Haenszel test and univariable conditional logistic regression for categorical and continuous variables, respectively. To identify factors associated with our composite outcome (intubation/ICU/death), we used the chi-square test, or Fisher exact test if the expected count was $<5$. Continuous variables were reported as medians and IQR, and compared using the Wilcoxon rank-sum test. We then performed multivariable conditional logistic regression to evaluate factors independently associated with our composite outcome, with the model including all variables $P \leq 0.2$ on univariable analysis. AI disease was included in the multivariable model a priori.

Kaplan-Meier analysis was performed when assessing the outcome of death alone, with the log-rank test used to measures differences. Multivariate Cox proportional hazards models were also used to characterize time to death, with adjustment of all included variables. All $P$ values are 2 -sided with an $\alpha$ level of 0.05 , and statistical analysis was performed with Stata
15.1 (StataCorp). The Institutional Review Board at Columbia University Medical Center approved this study (AAAS9860).

\section{RESULTS}

Baseline characteristics. We identified 62 patients with AI disorders and COVID-19 admitted to the hospital between March 1, 2020, and April 15, 2020 (Supplementary Figure 1, available from the authors on request). Of the AI disorders included (Supplementary Table 1), rheumatoid arthritis was the most represented with $16(25.8 \%)$ patients admitted, followed by sarcoidosis with 8 (12.9\%).

Among the 186 patients included in the analysis, there were no significant differences in BMI or smoking status between patients with AI disease and their non-AI disease age- and sex-matched controls (Table 1). Patients with AI disease were more likely to report White race/ethnicity versus patients without AI disease, who were more likely to report being Hispanic or Black $(P=0.01)$. Patients with AI disease were more likely to have at least 1 comorbidity and were more likely to have HTN $(75.8 \%$ vs $60.5 \%, P=0.03)$. Among the other comorbidities included, there were no significant differences between the prevalence of underlying respiratory diseases, DM, CKD, cardiac disease, or active malignancy.

When comparing transplantation history between the 2 groups, patients with AI disease had a significantly higher history of solid organ transplant compared to controls ( $16.1 \%$ vs $1.6 \%, P<0.01)$, with the majority of transplant patients $(75 \%)$ continuing home immunosuppressive medications as inpatients. As expected, a higher proportion of individuals with AI disease compared to controls were on chronic immunosuppression prior to admission $(66.1 \%$ vs $4.0 \%, P<0.01)$, with a complete list of the medications noted in Supplementary Table 2 (available from the authors on request). For those that were on chronic corticosteroids (CS), the majority (73.3\%) were on low-dose CS (budesonide, < $10 \mathrm{mg}$ prednisone). Of note, patients with AI disease were also more likely to be on long-term hydroxychloroquine (HCQ) therapy than their matched controls $(11.3 \%$ vs $0.0 \%, P<0.01$; Table 1$)$.

When assessing symptoms present on admission (Supplementary Table 3, available from the authors on request), there did not appear to be any significant differences in constitutional, neurologic, pulmonary, or gastrointestinal symptoms between the 2 populations. Additionally, there was no statistical difference in clinical presentation between cases and controls, as initial vital signs and laboratory values were similar (Table 1). However, during their hospital course, a greater proportion of patients with AI disease were given CS compared to controls (27.4\% vs $10.5 \%, P<0.01)$. The majority $(76.5 \%)$ of patients with AI disease receiving inpatient CS were continuing their home dose, in comparison to the non-AI disease group in which the majority (92.3\%) were newly initiating corticosteroids for COVID-19. There were no significant differences in any other administered inpatient treatments, including HCQ, azithromycin, or tocilizumab.

Median length of stay was not noted to be significantly longer in the AI group compared to the control group (7 vs 5 
Table 1. Univariable analysis of demographic information (age-/sex-matched) stratified by autoimmune disease in 186 hospitalized patients testing positive for SARS-CoV-2.

\begin{tabular}{|c|c|c|c|}
\hline & $\begin{array}{l}\text { No Autoimmune } \\
\text { Disease, } n=124\end{array}$ & $\begin{array}{c}\text { Autoimmune } \\
\text { Disease, } \mathrm{n}=62\end{array}$ & $P$ \\
\hline \multicolumn{4}{|l|}{ Age, yrs } \\
\hline $18-30$ & $6(4.8)$ & $3(4.8)$ & \\
\hline $31-44$ & $6(4.8)$ & $3(4.8)$ & \\
\hline $45-59$ & $32(25.8)$ & $16(25.8)$ & \\
\hline $60-74$ & $54(43.6)$ & $27(43.6)$ & \\
\hline$\geq 75$ & $26(21.0)$ & $13(21.0)$ & \\
\hline \multicolumn{4}{|l|}{ Sex } \\
\hline Male & $48(38.7)$ & $24(38.7)$ & \\
\hline Female & $76(61.3)$ & $38(61.3)$ & \\
\hline Race/ethnicity & & & 0.01 \\
\hline White & $26(21.0)$ & $24(38.7)$ & \\
\hline Hispanic & $61(49.2)$ & $17(27.4)$ & \\
\hline Black & $14(11.3)$ & $5(8.1)$ & \\
\hline Other/not listed & $23(18.6)$ & $16(25.8)$ & \\
\hline BMI & & & 0.29 \\
\hline$<18.5$ & $6(4.8)$ & $1(1.6)$ & \\
\hline $18.5-24.9$ & $18(14.5)$ & $10(16.1)$ & \\
\hline$\geq 25-29.9$ & $36(29.0)$ & $25(40.3)$ & \\
\hline $30-39.9$ & $38(30.7)$ & $18(29.0)$ & \\
\hline$\geq 40$ & $26(21.0)$ & $8(12.9)$ & \\
\hline \multicolumn{4}{|l|}{ Comorbidities } \\
\hline None & $32(25.8)$ & $8(12.9)$ & 0.03 \\
\hline HTN & $75(60.5)$ & $47(75.8)$ & 0.03 \\
\hline COPD/asthma/ILD & $20(16.1)$ & $14(22.6)$ & 0.28 \\
\hline DM2 & $38(30.7)$ & $14(22.6)$ & 0.26 \\
\hline CKD & $14(11.3)$ & $11(17.7)$ & 0.23 \\
\hline $\mathrm{CAD} / \mathrm{HF}$ & $25(20.2)$ & $15(24.2)$ & 0.54 \\
\hline Active cancer & $6(4.8)$ & $4(6.5)$ & 0.66 \\
\hline Smoking & & & 0.46 \\
\hline Never & $103(83.1)$ & $49(79.0)$ & \\
\hline Current & $4(3.5)$ & $1(1.6)$ & \\
\hline Former & $17(13.7)$ & $12(20.0)$ & \\
\hline Solid organ transplant & & & $<0.01$ \\
\hline No & $122(98.4)$ & $52(83.9)$ & \\
\hline Yes & $2(1.6)$ & $10(16.1)$ & \\
\hline \multicolumn{4}{|l|}{ Home medications } \\
\hline Immunosuppression & $5(4.0)$ & $41(66.1)$ & $<0.01$ \\
\hline Long-term HCQ & $0(0.0)$ & $7(11.3)$ & $<0.01$ \\
\hline ACEi/ARB & $33(26.6)$ & $21(33.9)$ & 0.31 \\
\hline Statin & $41(33.1)$ & $28(45.2)$ & 0.07 \\
\hline NSAID & $34(27.4)$ & $21(33.9)$ & 0.30 \\
\hline \multicolumn{4}{|l|}{ Inpatient medications } \\
\hline HCQ & $61(49.2)$ & $37(59.7)$ & 0.17 \\
\hline Azithromycin & $43(34.7)$ & $22(35.5)$ & 0.92 \\
\hline Tocilizumab & $5(4.0)$ & $2(3.2)$ & 0.79 \\
\hline Corticosteroids & $13(10.5)$ & $17(27.4)$ & $<0.01$ \\
\hline Remdesivir & $2(1.6)$ & $0(0.0)$ & \\
\hline \multicolumn{4}{|l|}{ Symptoms at admission } \\
\hline Constitutional & $96(89.7)$ & $46(86.8)$ & 0.50 \\
\hline Neurologic & $29(27.1)$ & $9(17.0)$ & 0.09 \\
\hline Pulmonary & $90(84.1)$ & $43(81.1)$ & 0.55 \\
\hline GI & $51(47.7)$ & $29(54.7)$ & 0.66 \\
\hline \multicolumn{4}{|l|}{ Initial vital signs } \\
\hline $\operatorname{Tmax}$ in $24 \mathrm{~h}$ & & & 0.16 \\
\hline$<38^{\circ} \mathrm{C}$ & $47(37.9)$ & $30(48.4)$ & \\
\hline$\geq 38^{\circ} \mathrm{C}$ & $77(62.1)$ & $32(51.6)$ & \\
\hline
\end{tabular}




\begin{tabular}{|c|c|c|c|}
\hline & $\begin{array}{l}\text { No Autoimmune } \\
\text { Disease, } n=124\end{array}$ & $\begin{array}{c}\text { Autoimmune } \\
\text { Disease, } \mathrm{n}=62\end{array}$ & $P$ \\
\hline Lowest $\mathrm{BP}$ in $24 \mathrm{~h}$, systolic & & & 0.90 \\
\hline$<100$ & $33(26.6)$ & $16(25.8)$ & \\
\hline $100-130$ & $80(64.5)$ & $37(59.7)$ & \\
\hline$>130$ & $11(8.9)$ & $9(14.5)$ & \\
\hline Admission HR & & & 0.07 \\
\hline$<100$ & $73(58.9)$ & $28(45.2)$ & \\
\hline$\geq 100$ & $51(41.1)$ & $34(54.8)$ & \\
\hline Admission RR & & & 0.12 \\
\hline$\leq 20$ & $96(77.4)$ & $41(66.1)$ & \\
\hline$>20$ & $28(22.6)$ & $21(33.9)$ & \\
\hline $\mathrm{O}_{2}$ support on ER presentation & & & 0.21 \\
\hline Room air & $105(84.7)$ & $48(77.4)$ & \\
\hline Nasal cannula & $11(8.9)$ & $10(16.1)$ & \\
\hline Nonrebreather & $7(5.7)$ & $3(4.8)$ & \\
\hline Intubation & $1(0.8)$ & $1(1.6)$ & \\
\hline \multicolumn{4}{|l|}{ Initial laboratory values, median (IQR) } \\
\hline $\mathrm{WBC}, / \mu \mathrm{L}$ & $7.0(5.2-9.3)$ & $7.3(5.5-10.4)$ & 0.18 \\
\hline Lymphocyte count, \% & $15.9(8.7-22.0)$ & $13.2(8.7-22.1)$ & 0.32 \\
\hline $\mathrm{ESR}, \mathrm{mm} / \mathrm{h}$ & $74(44.0-97.0)$ & $62(48.5-100)$ & 0.94 \\
\hline CRP, mg/L & $99.1(50.5-181.5)$ & $93.8(43.9-197.1)$ & 0.81 \\
\hline PCT, ng/mL & $0.2(0.1-0.5)$ & $0.2(0.12-0.52)$ & 0.41 \\
\hline IL-6, pg/mL & $26.8(5.0-96.9)$ & $16.0(5.0-68.9)$ & 0.17 \\
\hline Ferritin, ng/mL & $646.5(234.7-1039.0)$ & $526.8(233.3-807.5)$ & 0.14 \\
\hline D-dimer, ug/mL & $1.2(0.8-1.9)$ & $1.3(0.8-2.9)$ & 0.17 \\
\hline $\mathrm{LDH}, \mathrm{U} / \mathrm{L}$ & $398(285.0-556.0)$ & $395(257.0-516.0)$ & 0.30 \\
\hline $\mathrm{CK}, \mathrm{U} / \mathrm{L}$ & $117.0(71.0-300.0)$ & $119.5(70.0-294.0)$ & 0.36 \\
\hline LOS, days, median (IQR) & $5.0(1.0-10.0)$ & $7.0(3.0-12.0)$ & 0.26 \\
\hline Intubation & & & 0.47 \\
\hline No & $107(86.3)$ & $51(82.3)$ & \\
\hline Yes & $17(14.2)$ & $11(17.7)$ & \\
\hline ICU admission & & & 0.32 \\
\hline No & $107(86.3)$ & $50(80.7)$ & \\
\hline Yes & $17(13.7)$ & $12(19.4)$ & \\
\hline Death & & & 0.78 \\
\hline No & $104(83.9)$ & $53(85.5)$ & \\
\hline Yes & $20(16.1)$ & $9(14.5)$ & \\
\hline
\end{tabular}

Values are presented as $\mathrm{n}(\%)$ unless otherwise stated. ACEi: angiotensin-converting enzyme inhibitor; ARB: angiotensin II receptor blocker; $\mathrm{BP}$ : blood pressure; CAD: coronary artery disease; $\mathrm{CK}$ : creatine kinase; CKD: chronic kidney disease; COPD: chronic obstructive pulmonary disease; CRP: C-reactive protein; DM2: type 2 diabetes mellitus; ER: emergency room; ESR: erythrocyte sedimentation rate; GI: gastrointestinal; HCQ: hydroxychloroquine; HF: heart failure; HR: heart rate; HTN: hypertension; ICU: intensive care unit; ILD: interstitial lung disease; IL: interleukin; LDH: lactate dehydrogenase; LOS: length of stay; NSAID: nonsteroidal antiinflammatory drug; PCT: procalcitonin; RR: respiratory rate; Tmax: maximum temperature; WBC: white blood cell.

days, $P=0.26)$, even when only assessing the 141 patients alive and discharged during the study period ( 5 vs 4 days, $P=0.22$ ). A total of 29 deaths out of 186 patients were noted (15.6\%), with similar outcomes between the AI disease group and the controls, and no differences in intubation rates $(17.7 \%$ vs $13.7 \%$, $P=0.47)$, ICU admissions ( $19.4 \%$ vs $13.7 \%, P=0.32$ ), or rates of death $(14.5 \%$ vs $16.1 \%$; $P=0.78)$. Of note, only 1 patient was admitted to the ICU but not intubated, and all patients placed in hospice care during their admission died within the hospital.

Univariable and multivariable analysis of composite outcome (intubation/ICU/death). On univariable analysis, the presence of AI disease among patients hospitalized with COVID-19 was not associated with our composite outcome of intubation, ICU, or death $(P=0.63$; Table 2$)$. Similarly, we found that patients on long-term immunosuppression, including biologics, were also not at increased risk for adverse outcomes $(25.0 \%$ vs $23.9 \%$, $P=0.88)$. Predictors of adverse outcomes included older age $(P<0.01)$ and the presence of HTN $(P=0.04)$ or cardiovascular disease $(P=0.04)$. Conversely, having no comorbidities was associated with a lower risk of an adverse event $(P=0.04)$. Of note, inpatient treatment with HCQ $(P=0.02)$ or new initiation of a CS $(P=0.04)$ were associated with adverse outcomes. 
Table 2. Univariable analysis of adverse outcome (intubation/ICU/death) in all admitted SARS-CoV-2-positive patients.

\begin{tabular}{|c|c|c|c|}
\hline & $\begin{array}{c}\text { No Adverse } \\
\text { Outcome, } \mathrm{n}=140\end{array}$ & $\begin{array}{l}\text { Adverse Outcome, } \\
\qquad \mathrm{n}=46\end{array}$ & $P$ \\
\hline \multicolumn{4}{|l|}{ Age, yrs } \\
\hline $18-30$ & $9(6.4)$ & $0(0.0)$ & $<0.01$ \\
\hline $31-44$ & $8(5.7)$ & $1(2.2)$ & \\
\hline $45-59$ & $41(29.3)$ & $7(15.2)$ & \\
\hline $60-74$ & $62(44.3)$ & $19(41.3)$ & \\
\hline$\geq 75$ & $20(14.3)$ & $19(41.3)$ & \\
\hline \multicolumn{4}{|l|}{ Sex } \\
\hline Male & $53(37.9)$ & $19(41.3)$ & 0.68 \\
\hline Female & $87(62.1)$ & $27(58.7)$ & \\
\hline \multicolumn{4}{|l|}{ Race/ethnicity } \\
\hline White & $35(25.0)$ & $15(32.6)$ & 0.33 \\
\hline Hispanic & $64(45.7)$ & $14(30.4)$ & \\
\hline Black & $13(9.3)$ & $6(13.0)$ & \\
\hline Other/not listed & $28(20.0)$ & $11(23.9)$ & \\
\hline \multicolumn{4}{|l|}{ BMI } \\
\hline$<18.5$ & $4(2.9)$ & $3(6.5)$ & 0.28 \\
\hline $18.5-24.9$ & $18(12.9)$ & $10(21.7)$ & \\
\hline$\geq 25-29.9$ & $49(35.0)$ & $12(26.1)$ & \\
\hline $30-39.9$ & $45(32.1)$ & $11(23.9)$ & \\
\hline$\geq 40$ & $24(17.1)$ & $10(21.7)$ & \\
\hline \multicolumn{4}{|l|}{ Autoimmune disease } \\
\hline No & $92(65.7)$ & $32(69.6)$ & 0.63 \\
\hline Yes & $48(34.3)$ & $14(30.4)$ & \\
\hline \multicolumn{4}{|l|}{ Comorbidities } \\
\hline None & $35(25.0)$ & $5(10.9)$ & 0.04 \\
\hline HTN & $86(61.4)$ & $36(78.3)$ & 0.04 \\
\hline COPD/asthma/ILD & $27(19.3)$ & $7(15.2)$ & 0.54 \\
\hline $\mathrm{DM} 2$ & $37(26.4)$ & $15(32.6)$ & 0.42 \\
\hline CKD & $19(13.6)$ & $6(13.0)$ & 0.93 \\
\hline $\mathrm{CAD} / \mathrm{HF}$ & $25(17.9)$ & $15(32.6)$ & 0.04 \\
\hline Active cancer & $8(5.7)$ & $2(4.4)$ & 0.72 \\
\hline \multicolumn{4}{|l|}{ Smoking } \\
\hline Never & $119(85.0)$ & $33(71.7)$ & 0.09 \\
\hline Current & $3(2.1)$ & $2(4.4)$ & \\
\hline Former & $18(12.9)$ & $11(23.9)$ & \\
\hline \multicolumn{4}{|l|}{ Solid organ transplant } \\
\hline No & $130(92.9)$ & $44(95.7)$ & 0.50 \\
\hline Yes & $10(7.1)$ & $2(4.4)$ & \\
\hline \multicolumn{4}{|l|}{ Home medications } \\
\hline Immunosuppression & $35(25.0)$ & $11(23.9)$ & 0.88 \\
\hline Biologics & $10(7.1)$ & $1(2.2)$ & 0.22 \\
\hline $\mathrm{CS}$ & $21(15.0)$ & $9(19.6)$ & 0.45 \\
\hline Long-term HCQ & $6(4.3)$ & $1(2.2)$ & 0.45 \\
\hline $\mathrm{ACEi} / \mathrm{ARB}$ & $38(27.1)$ & $16(34.8)$ & 0.32 \\
\hline Statin & $51(36.4)$ & $18(39.1)$ & 0.74 \\
\hline NSAID & $40(28.6)$ & $15(32.6)$ & 0.60 \\
\hline \multicolumn{4}{|l|}{ Inpatient medications } \\
\hline HCQ & $67(47.9)$ & $31(67.4)$ & 0.02 \\
\hline Azithromycin & $46(32.9)$ & $19(41.3)$ & 0.30 \\
\hline Tocilizumab & $3(2.1)$ & $4(8.7)$ & 0.06 \\
\hline $\mathrm{CS}^{*}$ & $6(4.3)$ & $6(13.0)$ & 0.04 \\
\hline Remdesivir & $0(0.0)$ & $2(4.4)$ & 0.06 \\
\hline \multicolumn{4}{|l|}{ Symptoms at admission } \\
\hline Constitutional & $115(89.8)$ & $27(84.4)$ & 0.38 \\
\hline Neurologic & $34(26.6)$ & $4(12.5)$ & 0.07 \\
\hline Pulmonary & $108(84.4)$ & $25(78.1)$ & 0.40 \\
\hline GI & $68(53.1)$ & $12(37.5)$ & 0.11 \\
\hline
\end{tabular}




\begin{tabular}{|c|c|c|c|}
\hline & $\begin{array}{c}\text { No Adverse } \\
\text { Outcome, } n=140\end{array}$ & $\begin{array}{l}\text { Adverse Outcome, } \\
\qquad \mathrm{n}=46\end{array}$ & $P$ \\
\hline \multicolumn{4}{|l|}{ Initial vital signs } \\
\hline \multicolumn{4}{|l|}{$\operatorname{Tmax}$ in $24 \mathrm{~h}$} \\
\hline$<38^{\circ} \mathrm{C}$ & $55(39.3)$ & $22(47.8)$ & \multirow[t]{2}{*}{0.31} \\
\hline$\geq 38^{\circ} \mathrm{C}$ & $85(60.7)$ & $24(52.2)$ & \\
\hline \multicolumn{4}{|c|}{ Lowest $\mathrm{BP}$ in $24 \mathrm{~h}$, systolic } \\
\hline$<100$ & $29(20.7)$ & $20(43.5)$ & \multirow[t]{3}{*}{$<0.01$} \\
\hline $100-130$ & $94(67.1)$ & $23(50.0)$ & \\
\hline$>130$ & $17(12.1)$ & $3(6.5)$ & \\
\hline \multicolumn{4}{|l|}{ Admission HR } \\
\hline$<100$ & $77(55.0)$ & $24(52.2)$ & \multirow[t]{2}{*}{0.74} \\
\hline$\geq 100$ & $63(45.0)$ & $22(47.8)$ & \\
\hline \multicolumn{4}{|l|}{ Admission RR } \\
\hline$\leq 20$ & $113(80.7)$ & $24(52.2)$ & \multirow[t]{2}{*}{$<0.01$} \\
\hline$>20$ & $27(19.3)$ & $22(47.8)$ & \\
\hline \multicolumn{4}{|c|}{$\mathrm{O}_{2}$ support on ER presentation } \\
\hline Room air & $127(90.7)$ & $26(56.5)$ & \multirow[t]{4}{*}{$<0.01$} \\
\hline Nasal cannula & $12(8.6)$ & $9(19.6)$ & \\
\hline Nonrebreather & $1(0.7)$ & $9(19.6)$ & \\
\hline Intubation & $0(0.0)$ & $2(4.4)$ & \\
\hline \multicolumn{4}{|c|}{ Initial lab values, median (IQR) } \\
\hline WBC, $/ \mu \mathrm{L}$ & $6.7(5.1-9.1)$ & $8.4(5.8-13.9)$ & $<0.01$ \\
\hline Lymphocyte count, \% & $16.6(10.0-23.1)$ & $10.8(7.0-18.6)$ & $<0.01$ \\
\hline $\mathrm{ESR}, \mathrm{mm} / \mathrm{h}$ & $65.0(43.0-96.5)$ & $30.7(55.5-102.5)$ & 0.06 \\
\hline $\mathrm{CRP}, \mathrm{mg} / \mathrm{L}$ & $88.9(41.7-173.3)$ & $152.7(92.4-222.2)$ & $<0.01$ \\
\hline PCT, ng/mL & $0.14(0.1-0.3)$ & $0.48(0.2-1.2)$ & $<0.01$ \\
\hline IL-6, pg/mL & $15.0(5.0-41.0)$ & $75.2(16.0-164.7)$ & $<0.01$ \\
\hline Ferritin, $\mathrm{ng} / \mathrm{mL}$ & $589.0(217.3-972.0)$ & $625.8(374.3-1117.0)$ & 0.31 \\
\hline D-dimer, ug/mL & $1.06(0.8-1.7)$ & $1.88(0.9-3.2)$ & $<0.01$ \\
\hline $\mathrm{LDH}, \mathrm{U} / \mathrm{L}^{\circ}$ & $373.5(274.0-514.0)$ & $458.0(333.0-661.0)$ & 0.02 \\
\hline $\mathrm{CK}, \mathrm{U} / \mathrm{L}$ & $110.5(62.0-285.0)$ & $146.0(77.0-403.0)$ & 0.17 \\
\hline
\end{tabular}

${ }^{*}$ Excluding patients continued on home CS dosing. Values are presented as n (\%) unless otherwise stated. ACEi: angiotensin-converting enzyme inhibitor; ARB: angiotensin II receptor blocker; BP: blood pressure; CAD: coronary artery disease; $\mathrm{CK}$ : creatine kinase; CKD: chronic kidney disease; COPD: chronic obstructive pulmonary disease; CRP: C-reactive protein; CS: corticosteroids; DM2: type 2 diabetes mellitus; ER: emergency room; ESR: erythrocyte sedimentation rate; GI: gastrointestinal; HCQ: hydroxychloroquine; HF: heart failure; HR: heart rate; HTN: hypertension; ICU: intensive care unit; ILD: interstitial lung disease; IL: interleukin; LDH: lactate dehydrogenase; NSAID: nonsteroidal antiinflammatory drug; PCT: procalcitonin; RR: respiratory rate; Tmax: maximum temperature; WBC: white blood cell.

Patients with a higher incidence of adverse outcomes also had higher median white blood cell count, procalcitonin level, and proinflammatory laboratory profiles, including higher C-reactive protein, interleukin 6 levels, and D-dimer, but were noted to have a lower lymphocyte count on admission $(P<0.01$ for all mentioned laboratory values; Table 2).

On multivariable analysis using conditional logistic regression, the presence of AI disease was not a significant risk factor for adverse outcomes among hospitalized COVID-19 patients, when adjusting for smoking and comorbidities, as compared to age- and sex-matched controls $\left(\mathrm{OR}_{\text {adj }} 0.79,95 \%\right.$ CI 0.37-1.67, $P=0.53$; Table 3 ). On sensitivity analysis, when including BMI and race/ethnicity in our model, we similarly found that AI disease was not associated with adverse outcomes $\left(\mathrm{OR}_{\text {adj }} 0.67\right.$, 95\% CI 0.25-1.80, $P=0.43$; data not shown).

Coxproportionalhazardsassessingtime to death. On Kaplan-Meier
Table 3. Conditional logistic regression of adverse outcome (intubation/ ICU/death) in all patients admitted with COVID-19.

\begin{tabular}{lc}
\hline & Adjusted OR $(95 \% \mathrm{CI})^{*}$ \\
\hline $\begin{array}{c}\text { Autoimmune disease } \\
\text { No }\end{array}$ & $($ Ref. $)$ \\
Yes & $0.79(0.37-1.67)$ \\
Comorbidities & $($ Ref. $)$ \\
$\quad$ None & $1.44(0.37-5.62)$ \\
1 & $1.74(0.51-6.00)$ \\
$\geq 2$ & $($ Ref. $)$ \\
Smoking & $3.06(0.22-43.61)$ \\
Never & $5.21(0.87-31.40)$ \\
Current & \\
\hline Former & \\
\hline
\end{tabular}

* Adjusted for all variables in the table. COVID-19: coronavirus disease 2019. 
analysis, although there was increased time to death among patients with AI, these results were not statistically significant (log-rank $=0.47$; Figure 1), with a HR of $0.73(95 \%$ CI $0.33-1.63, P=0.45$; Table 4 ) when adjusting for smoking and comorbidities. On sensitivity analysis, when including BMI and race/ethnicity in our model, we found similar results $\left(\mathrm{HR}_{\mathrm{adj}} 0.45\right.$, 95\% CI 0.18-1.13, $P=0.09$ ). Additionally, when only including the 170 patients discharged or deceased, we observed similar findings (log-rank: 0.08; Supplementary Figure 2, available from the author on request).

\section{DISCUSSION}

In our study, we found that patients with AI disease hospitalized with COVID-19 had a similar constellation of presenting symptoms, vital signs, and lab values, and had similar lengths of stay compared to matched controls. Further, AI disease did not increase the risk of adverse outcomes and was not associated with an increased hazard of death when adjusting for relevant clinical variables.

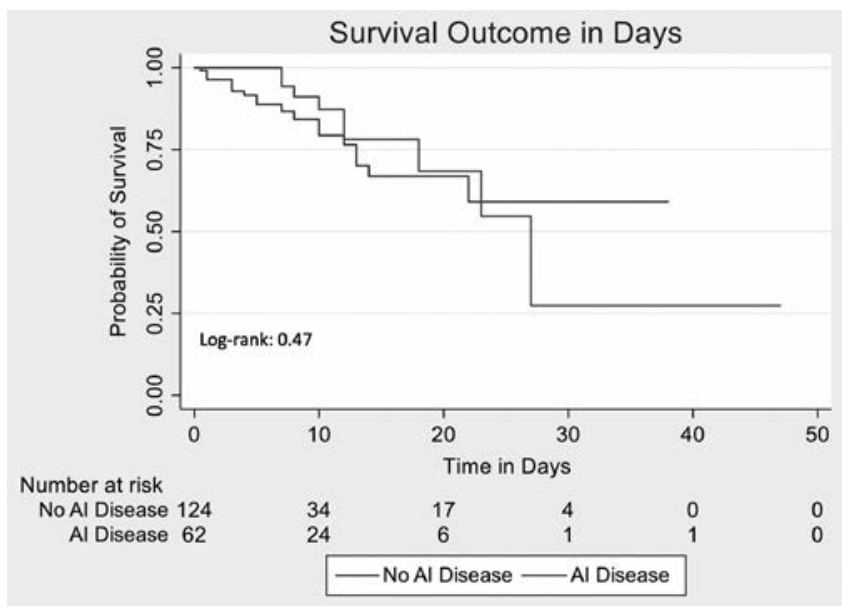

Figure 1. Kaplan-Meier curve examining death, stratified by the presence or absence of autoimmune disease in all 186 patients, with 16 patients censored as of April 29, 2020. AI: autoimmune.

Table 4. Cox proportional hazards examining time to death among the 186 included patients.

Adjusted HR (95\% CI)*

\begin{tabular}{lc}
\hline Autoimmune disease & \\
No & (Ref.) \\
Yes & $0.73(0.33-1.63)$ \\
Comorbidities & (Ref.) \\
None & $1.08(0.31-3.75)$ \\
1 & $0.93(0.31-2.84)$ \\
$\geq 2$ & $($ Ref.) \\
Smoking & $1.40(0.18-10.6)$ \\
Never & $1.86(0.83-4.19)$ \\
Current & \\
Former &
\end{tabular}

* Adjusted for all variables in the table.
In an initial study by Haberman, et al, 86 COVID-19 (59 confirmed and 27 suspected) patients with IMID were found to have a similar incidence of hospitalization compared to the general New York City population; however, no comparator arm was used to make inferences specific to AI disease and only 14 patients were hospitalized ${ }^{20}$. More recently, a study by D'Silva, et al built upon this by assessing 52 patients with rheumatic diseases and comparing them to 104 matched controls, though only a small number were hospitalized $(n=23)$, which limited the assessment of in-hospital outcomes ${ }^{11}$.

In our present study, we specifically looked to assess the outcomes of hospitalized COVID-19 patients with and without AI disease. As such, we analyzed 62 hospitalized COVID-19positive AI disease cases and compared them to 124 hospitalized age- and sex-matched COVID-19-positive controls without AI disease (all RT-PCR confirmed). On baseline characteristics, we found that individuals with AI disease tended to have more comorbidities than their matched controls, particularly HTN and a history of solid organ transplant. Further, a high proportion of patients with AI were taking chronic immunosuppressive medications (66.1\%). Although immunosuppression is currently being used as a potential therapy for COVID-1921, data looking at outcomes among patients on chronic immunosuppression remains sparse. In a recent study of 90 solid organ transplant patients on chronic immunosuppression, Pereira, et al found that transplant recipients who developed COVID-19 overall had a severe disease course ${ }^{22}$. However, a small study by Cavagna, et al found that calcineurin inhibitors were associated with a mild course of disease ${ }^{23}$. Within our study, we also reassuringly found that long-term immunosuppression, including in patients with AI, was not significantly associated with adverse outcomes. When long-term immunosuppression was stratified by high and low doses, there was also no difference in adverse outcomes $^{24}$. Additionally, we found that outpatient CS use was not associated with adverse outcomes. This is in line with prior studies showing that among patients with COVID-19 ill enough to become hospitalized, home CS use may no longer be a risk factor for severe outcomes ${ }^{25}$. When only examining patients taking biologics, similar results were seen. This is in accordance with recently published data from international registries of patients with rheumatic and inflammatory bowel diseases, which found that biologics were not associated with increased severity of disease ${ }^{26}$.

On admission, individuals with AI disorders had similar symptoms, vital signs, and laboratory values, including those suggestive of cytokine storm, compared to their matched controls; this is analogous to findings observed by D'Silva, et $a l^{11}$. Patients with AI disease, however, were more likely to receive CS during their hospital course, which was largely driven by the continuation of their home CS regimens. Of note, we did find that inpatient initiation of CS or HCQ was associated with adverse outcomes, though this is likely a marker of disease severity (higher acuity patients were more likely to receive these medications).

When assessing disease outcomes, recent reports have been conflicting, with some studies noting a higher risk of respiratory 
failure among patients with rheumatic disease hospitalized with COVID-19 ${ }^{11,14,27}$. In our study, which represents one of the largest inpatient samples of AI disease patients to date, we observed no differences in the risk of intubation, ICU admission, or death between patients with AI disease and matched controls. Further, after adjusting for smoking and comorbidities, as well as the clinically relevant variables of race/ethnicity and $\mathrm{BMI}^{8,11,28,29,30,31,32}$, we found that patients with AI disease were not at higher risk for the composite outcome of intubation, ICU admission, or death compared to controls.

Although some studies have suggested a higher risk of respiratory failure in patients with autoimmune disease, most have not observed an increase in overall mortality ${ }^{11,14}$. In accordance with this, when assessing time to death, we found that patients with AI disease did not have an increased HR. Additionally, we observed no significant difference in length of stay between patients with AI disease and controls, which has been similarly reported in these prior studies. As such, our findings provide reassurance that hospitalized COVID-19 patients with AI disorders, including those on immunosuppressive therapy, do not appear to be at significantly higher risk for adverse clinical outcomes compared to hospitalized COVID-19-positive controls without AI disease. In addition, we did not observe significant differences in clinical presentation, suggesting that hospitalized COVID-19 patients with AI disease may not be at increased risk for cytokine storm. While this may be a result of chronic immunosuppressive therapy, further investigation is needed.

Our study has limitations. Given our available sample size, we had sufficient power to detect an increased risk of adverse outcomes with an $\mathrm{OR} \geq 1.72$. It is possible that a more modest risk could be present, though our findings suggest a nonsignificant trend towards a lower overall risk. Although we included a broad range of AI disorders, we were unable to perform stratified analyses by each disorder due to the limited number of patients with AI hospitalized with COVID-19. As such, the heterogeneity of these disorders may mask differential risks between them. Nonetheless, given the rarity of these disorders in the hospitalized COVID-19 population, and the critical need for data to help guide clinical practice in the context of this pandemic, we believe these analyses provide valuable insights into the management of individuals with AI conditions diagnosed with COVID-19. Additionally, because many patients did not have race/ethnicity listed in the EHR, we were unable to accurately match on this variable. On univariable analysis, however, race/ ethnicity did not appear to be an independent predictor of our primary outcome. Finally, this was a single-center study, which might limit the generalizability of our results.

This is one of the largest inpatient cohorts to date that examines the association of AI disorders with clinical outcomes of COVID-19. A particular strength is the use of a matched cohort design for the execution of our study. The age- and sex-matched controlled design reduces bias, since age and sex play important roles in the development of many AI disorders and in outcomes related to COVID-19. Thus, by ensuring an equal distribution of these variables among both groups, we were able to mitigate their potential effects as confounders. In addition, we included a secondary analysis which allowed us to further evaluate the association between AI disease and time to death. Finally, all data for our analysis were manually extracted and reviewed for accuracy (including the distinction between outpatient HCQ initiation for COVID-19 vs long-term use as a treatment agent for underlying AI disorders), which helps ensure the validity of this large dataset. Multivariable analysis was then performed in order to adjust for factors associated with our composite outcome.

In conclusion, we found that among patients hospitalized with COVID-19, individuals with AI disease, and those on chronic immunosuppressive therapy did not have an increased risk of adverse events, such as ICU admission, intubation, and death. Additional research is needed to better characterize the management of individuals with AI conditions in the context of COVID-19, particularly with regard to the use of additional immunosuppression in the inpatient setting.

\section{ACKNOWLEDGMENT}

The authors thank the many employees of NewYork-Presbyterian Hospital/ Columbia University Medical Center for their thoughtful care of these patients, as well as the patients themselves for their trust and participation in their care.

\section{REFERENCES}

1. Coronaviridae Study Group of the International Committee on Taxonomy of Viruses. The species Severe acute respiratory syndrome-related coronavirus: classifying $2019-\mathrm{nCoV}$ and naming it SARS-CoV-2. Nat Microbiol 2020;5:536-44.

2. Richardson S, Hirsch JS, Narasimhan M, Crawford JM, McGinn T, Davidson KW, et al. Presenting characteristics, comorbidities, and outcomes among 5700 patients hospitalized with COVID-19 in the New York City area. JAMA 2020;323:2052-9.

3. Ruan Q, Yang K, Wang W, Jiang L, Song J. Clinical predictors of mortality due to COVID-19 based on an analysis of data of 150 patients from Wuhan, China. Intensive Care Med 2020;46:846-8.

4. Wang D, Hu B, Hu C, Zhu F, Liu X, Zhang J, et al. Clinical characteristics of 138 hospitalized patients with 2019 novel coronavirus-infected pneumonia in Wuhan, China. JAMA 2020;323:1061-9.

5. Zhou F, Yu T, Du R, Fan G, Liu Y, Liu Z, et al. Clinical course and risk factors for mortality of adult inpatients with COVID-19 in Wuhan, China: a retrospective cohort study. Lancet 2020; 395:1054-62.

6. Li X, Xu S, Yu M, Wang K, Tao Y, Zhou Y, et al. Risk factors for severity and mortality in adult COVID-19 inpatients in Wuhan. J Allergy Clin Immunol 2020;146:110-8.

7. Huang C, Wang Y, Li X, Ren L, Zhao J, Hu Y, et al. Clinical features of patients infected with 2019 novel coronavirus in Wuhan, China. Lancet 2020;395:497-506.

8. Cummings MJ, Baldwin MR, Abrams D, Jacobson SD, Meyer BJ, Balough EM, et al. Epidemiology, clinical course, and outcomes of critically ill adults with COVID-19 in New York City: a prospective cohort study. Lancet 2020;395:1763-70.

9. Aggarwal S, Garcia-Telles N, Aggarwal G, Lavie C, Lippi G, Henry BM. Clinical features, laboratory characteristics, and outcomes of patients hospitalized with coronavirus disease 2019 (COVID-19): early report from the United States. Diagnosis 2020;7:91-6.

10. Zangrillo A, Beretta L, Scandroglio AM, Monti G, Fominskiy E, Colombo $S$, et al. Characteristics, treatment, outcomes and cause of 
death of invasively ventilated patients with COVID-19 ARDS in Milan, Italy. Crit Care Resusc 2020;22:200-11.

11. D'Silva KM, Serling-Boyd N, Wallwork R, Hsu T, Fu X, Gravallese EM, et al. Clinical characteristics and outcomes of patients with coronavirus disease 2019 (COVID-19) and rheumatic disease: a comparative cohort study from a US 'hot spot'. Ann Rheum Dis 2020;79:1156-62.

12. Gianfrancesco M, Hyrich KL, Al-Adely S, Carmona L, Danila MI, Gossec L, et al. Characteristics associated with hospitalisation for COVID-19 in people with rheumatic disease: data from the COVID-19 Global Rheumatology Alliance physician-reported registry. Ann Rheum Dis 2020;79:859-66.

13. Gianfrancesco MA, Hyrich KL, Gossec L, Strangfeld A, Carmona L, Mateus EF, et al. Rheumatic disease and COVID-19: initial data from the COVID-19 Global Rheumatology Alliance provider registries. Lancet Rheumatol 2020;2:e250-3.

14. Ye C, Cai S, Shen G, Guan H, Zhou L, Hu Y, et al. Clinical features of rheumatic patients infected with COVID-19 in Wuhan, China. Ann Rheum Dis 2020;79:1007-13.

15. Emmi G, Bettiol A, Mattioli I, Silvestri E, Di Scala G, Urban $\mathrm{ML}$, et al. SARS-CoV-2 infection among patients with systemic autoimmune diseases. Autoimmun Rev 2020;19: 102575.

16. Freites Nuñez DD, Leon L, Mucientes A, Rodriguez-Rodriguez L, Font Urgelles J, Madrid García A, et al. Risk factors for hospital admissions related to COVID-19 in patients with autoimmune inflammatory rheumatic diseases. Ann Rheum Dis 2020;79:1393-9.

17. Ungaro RC, Brenner EJ, Gearry RB, Kaplan GG, Kissous-Hunt M, Lewis JD, et al. Effect of IBD medications on COVID-19 outcomes: results from an international registry. Gut 2020 Oct 20 (E-pub ahead of print).

18. Mehta P, McAuley DF, Brown M, Sanchez E, Tattersall RS, Manson JJ; HLH Across Speciality Collaboration UK. COVID-19: consider cytokine storm syndromes and immunosuppression. Lancet 2020;395:1033-4.

19. Wang T, Du Z, Zhu F, Cao Z, An Y, Gao Y, et al. Comorbidities and multi-organ injuries in the treatment of COVID-19. Lancet 2020;395:e52.

20. Haberman R, Axelrad J, Chen A, Castillo R, Yan D, Izmirly P, et al. Covid-19 in immune-mediated inflammatory diseases - case series from New York. N Engl J Med 2020;383:85-8.

21. Zhang C, Wu Z, Li JW, Zhao H, Wang GQ. The cytokine release syndrome of severe COVID-19 and interleukin-6 receptor (IL-6R) antagonist tocilizumab may be the key to reduce the mortality. Int J Antimicrob Agents 2020;55:105954.
22. Pereira MR, Mohan S, Cohen DJ, Husain SA, Dube GK, Ratner LE, et al. COVID-19 in solid organ transplant recipients: initial report from the US epicenter. Am J Transplant 2020;20:1800-8.

23. Cavagna L, Seminari E, Zanframundo G, Gregorini M, Di Matteo A, Rampino T, et al. Calcineurin inhibitor based immunosuppression and COVID-19: results from a multidisciplinary cohort of patients in Northern Italy. Microorganisms 2020;8:977.

24. Pileggi GS, Da Mota LM, Kakehasi AM, De Souza AW, Rocha A, de Melo AK, et al. Brazilian recommendations on the safety and effectiveness of the yellow fever vaccination in patients with chronic immune-mediated inflammatory diseases. Adv Rheumatol 2019;59:17.

25. Pablos JL, Galindo M, Carmona L, Lledó A, Retuerto M, Blanco R, et al. Clinical outcomes of hospitalised patients with COVID-19 and chronic inflammatory and autoimmune rheumatic diseases: a multicentric matched cohort study. Ann Rheum Dis 2020; 70:1544-9.

26. Brenner EJ, Ungaro RC, Gearry RB, Kaplan GG, Kissous-Hunt $\mathrm{M}$, Lewis JD, et al. Corticosteroids, but not TNF antagonists, are associated with adverse COVID-19 outcomes in patients with inflammatory bowel diseases: results from an international registry. Gastroenterology 2020;159:481-91.

27. Rodríguez-Lago I, Ramírez de la Piscina P, Elorza A, Merino O, Ortiz de Zárate J, Cabriada JL. Characteristics and prognosis of patients with inflammatory bowel disease during the SARS-CoV-2 pandemic in the Basque Country (Spain). Gastroenterology 2020;159:781-3.

28. Lighter J, Phillips M, Hochman S, Sterling S, Johnson D, Francois $\mathrm{F}$, et al. Obesity in patients younger than 60 years is a risk factor for Covid-19 hospital admission. Clin Infect Dis 2020;71:896-7.

29. Goyal P, Choi JJ, Pinheiro LC, Schenck EJ, Chen R, Jabri A, et al. Clinical characteristics of Covid-19 in New York City. N Engl J Med 2020;382:2372-4.

30. Raifman MA, Raifman JR. Disparities in the population at risk of severe illness from COVID-19 by race/ethnicity and income. Am J Prev Med 2020;59:137-9.

31. Laurencin CT, McClinton A. The COVID-19 pandemic: a call to action to identify and address racial and ethnic disparities. J Racial Ethn Health Disparities 2020;7:398-402.

32. Kakkar DN, Dunphy DJ, Raza DM. Ethnicity profiles of COVID-19 admissions and outcomes. J Infect 2020; 81:e110-1. 